\title{
Estudo da influência do estágio de lactação na concentração dos principais ácidos graxos de lactante com bebê nascido a termo
}

Study of the influence of the lactation stage on the concentration of the main fatty acids in lactating women with full-term infants

Estudio de la influencia de la etapa de lactancia en la concentración de los principales ácidos grasos en mujeres lactantes con recién nacidos a término

\section{Resumo}

O presente estudo tem como objetivo avaliar a influência dos estágios de lactação (colostro, transição e maduro) na composição dos principais ácidos graxos e somatórios de ácidos graxos no leite humano de lactante com bebê nascido a termo. Para isso foi coletado de forma individualizada, no mesmo período do dia, e após 1 hora da mama ser esvaziada, por meio de ordenha manual cerca de $5 \mathrm{ml}$ de leite humano, em seguida foi realizada a composição de ácidos graxos por meio de cromatografia em fase gasosa acoplado a detector de ionização em chama (CG-DIC). O ácido graxo encontrado majoritariamente para o colostro foi o ácido linoleico (18:2n-6), já para os leites de transição e maduro foi o ácido oleico (18:1n-9), além disso, todos os ácidos graxos essenciais e estritamente essenciais foram identificados e quantificados nas três fases. Foi possível observar que o estágio de lactação influencia de forma direta na composição de ácido graxo, sendo que o leite colostro é o que apresenta maior distinção entre as fases de lactação, e o leite de transição e o leite maduro tem mais similaridade entre si.

Palavras-chave: Leite humano; Lactação; Ácido graxo; CG-DIC.

\section{Abstract}

The present study aims to evaluate the influence of the stages of lactation (colostrum, transition and mature) on the composition of the main fatty acids and sums of fatty acids in human milk from lactating women with full-term infants. For this, approximately $5 \mathrm{ml}$ of human milk was collected individually, in the same period of the day, and 
after 1 hour of the breast being emptied, through manual milking, then the fatty acid composition was performed by means of chromatography in gas phase coupled to flame ionization detector (CG-DIC). The fatty acid found mostly for colostrum was linoleic acid (18:2n-6), whereas for transition and mature milk it was oleic acid (18:1n-9), in addition to all essential fatty acids and strictly essential were identified and quantified in the three phases. It was possible to observe that the lactation stage directly influences the fatty acid composition, with colostrum milk being the one with the greatest distinction between the lactation phases, and transitional milk and mature milk having more similarity to each other.

Keywords: Human milk; Lactation; Fatty acid; CG-FID.

\section{Resumen}

El presente estudio tiene como objetivo evaluar la influencia de las etapas de la lactancia (calostro, transición y madurez) sobre la composición de los principales ácidos grasos y sumas de ácidos grasos en la leche materna de mujeres lactantes con recién nacidos a término. Para ello, se recolectaron aproximadamente $5 \mathrm{ml}$ de leche materna individualmente, en el mismo período del día, y luego de 1 hora de vaciado de la mama, mediante ordeño manual, luego se realizó la composición de ácidos grasos mediante cromatografía en fase gaseosa acoplada. al detector de ionización de llama (CG-DIC). El ácido graso encontrado mayoritariamente para el calostro fue el ácido linoleico (18: 2n-6), mientras que para la leche de transición y madura fue el ácido oleico (18: 1n-9), además de todos los ácidos grasos esenciales y estrictamente esenciales se identificaron y cuantificaron en las tres fases. Se pudo observar que la etapa de lactancia influye directamente en la composición de ácidos grasos, siendo la leche de calostro la que presenta mayor distinción entre las fases de lactancia, y la leche de transición y la leche madura teniendo más similitud entre sí. Palabras clave: Leche materna; Lactancia; Ácidos grasos; CG-DIC.

\section{Introdução}

O leite humano (LH) é um alimento caracterizado pelo equilíbrio quantitativo e qualitativo de nutrientes, essenciais para um crescimento saudável de lactentes (Van Gysel, Cossey, Fieuws, \& Schuermans, 2012). A Academia Americana de Pediatria recomenda que o LH seja fonte exclusiva de nutrição para lactentes até os seis primeiros meses de vida (Johnston, Landers, Noble, Szucs, \& Viehmann, 2012), fornecendo nutrientes e componentes bioativos, que auxiliam no desenvolvimento do organismo infantil, bem como na proteção contra doenças gastrointestinais, doenças respiratórias, redução de doenças crônicas como o diabetes tipo 1 e 2, auxiliando ainda, na adaptação ao ambiente externo do útero da mãe (Prell \& Koletzko, 2016; Binns, Lee, \& Low, 2016).

Sua classificação mais usual é de acordo com o tempo de lactação da mãe, sendo classificados em LH colostro, LH de transição e LH maduro. O LH colostro tem aparência ligeiramente amarelada e consistência mais líquida. Este leite contém os mesmos nutrientes que o LH maduro, porém, com mais proteínas e menos gordura e é considerado do primeiro ao sétimo dia após o parto. O LH transição é considerado do oitavo ao décimo quarto dia apresenta maior volume por mamada; quando comparado ao LH colostro, há alterações nutricionais graduais entre as características de colostro até o maduro, como a diminuição dos índices de proteína e aumento de gorduras e carboidratos. E o LH maduro é considerado a partir do décimo quinto dia e possui aparência mais esbranquiçada, é mais consistente que os leites anteriores (Ballard \& Morrow, 2013; Euclydes, 2005; Demmelmair \& Koletzko; 2018; Rydlewski et al., 2019).

Cerca de $98 \%$ da gordura do LH consiste em lipídios neutros, triacilglicerol (TAG), contendo diferentes ácidos graxos (AGs,), com cadeias laterais associadas localizadas nas posições Sn-1, Sn-2 e Sn-3, sendo que o restante é constituído de fosfolipídios e esteróis. O posicionamento desses AGs resulta em TAGs únicos, e essas estruturas afetam a eficiência da digestão e absorção de lipídios (Vyssotski, Bloor, Lagutin, Wong, \& Williams, 2015; Zhao et al., 2018; Wang et al., 2020). Os TAGs são conhecidos como a principal fonte de energia para os lactentes.

Apesar da abundância relativamente baixa de glicerofosfolípidos, esfingolípidos e glicolípidos, estes constituintes representam os componentes predominantes na membrana do glóbulo de gordura do leite, com um papel significativo na saúde infantil, associados ao neurodesenvolvimento, desenvolvimento cognitivo e aos efeitos protetores do LH (Bourlieu \& Michalski, 2015; Gurnida, Rowan, Idjradinata, Muchtadi, \& Sekarwana, 2012). 
$\mathrm{O}$ ácido graxo saturado majoritário encontrado no leite humano é o ácido palmítico (P, 16:0), geralmente o ácido palmítico encontra-se na posição central da molécula de TAG, sendo que dessa forma ele é facilmente absorvido, resultando em vários benefícios ao lactente, como melhora no desconforto intestinal, diminuição de cólicas, e apresenta também influência nos níveis de anandamida que apresenta efeito analgésico (Mehrotra, Sehgal, \& Bangale, 2019, Demmelmair \& Koletzko, 2018, Rydlewski et al., 2019).

Diante do exposto o objetivo do presente estudo foi de avaliar a influência dos estágios de lactação (colostro, transição e maduro) na composição dos principais ácidos graxos e somatórios de ácidos graxos no leite humano de lactantes com bebê nascido a termo.

\section{Metodologia}

\subsection{Amostragem}

O presente estudo estudo trata-se de uma pesquisa laboratorial e cientifica (Pereira, Shitsuka, Parreira, \& Shitsuka, 2018). Foi aprovado pelo comitê de ética em pesquisa pelo processo 2.797 .476 da Universidade Estadual de Maringá. Foram coletadas as amostras de leite humano das fases colostro, transição e maduro, de uma lactente, sempre coletado as 11 horas da manhã, após esvaziar a mama 1 hora anteriormente, a coleta do leite foi por meio de ordenha manual. Após a coleta, as amostras de LH foram homogeneizadas e armazenadas em recipiente de polietileno, em temperatura de $-18^{\circ} \mathrm{C}$, até o momento da análise.

\subsection{Extração lípidica}

Os lipídios foram extraídos de acordo com Folch, Lees, e Sloane-Stanley (1957). Pipetou-se $1 \mathrm{~mL}$ de leite humano, adicionando nesta os $20 \mathrm{~mL}$ do solvente (1:20) e razão clorofórmio: metanol (2:1) e agitou-se por 15 min. em agitador magnético. Ao término, centrifugou-se a amostra por $5 \mathrm{~min}$. em uma centrífuga refrigerada a $25{ }^{\circ} \mathrm{C}$ e $6000 \mathrm{rpm}$ para retirar a proteína precipitada. No segundo momento, adicionou-se $4 \mathrm{~mL}$ de solução de cloreto de sódio $(\mathrm{NaCl}) \quad 0,9 \%$, agitou-se a solução por $5 \mathrm{~min}$. e centrifugou-se (na mesma condição que a etapa anterior) para ocorrer a separação das fases.

A fase inferior foi coletada em um balão de $250 \mathrm{~mL}$, previamente pesado, e o solventeevaporado a $37{ }^{\circ} \mathrm{C}$ utilizando um rota evaporador rotativo.

\subsection{Esterificação}

Seguiu a metodologia proposta pela ISO 12966/2017, na qual, em um tubo de centrífuga, 100 mg de amostra lipídica são adicionadas a $2 \mathrm{~mL}$ de heptano, agitando por $2 \mathrm{~min}$. em vortex, em seguida, foram adicionados $3 \mathrm{~mL}$ do reagente esterificante hidróxido de potássio $(\mathrm{KOH}) 2 \mathrm{~mol} / \mathrm{L} \mathrm{em}$ metanol. Essa solução foi agitada por $3 \mathrm{~min}$. em vortex novamente, e levada à geladeira por 24 horas para separação de fases, sendo a fase superior analisada em um cromatógrafo a gás acoplado a um detector de ionização em chama (CG-DIC).

\subsection{Análise de composição em ácidos graxos}

As análises de ésteres metílicos de ácidos graxos (EMAGs) foram realizadas em cromatografia em fase gasosa (CG) da Thermo Scientific (Trace Ultra 3300, Waltham, Estados Unidos) com detector de ionização em chama (DIC), coluna capilar CP-7420 (100,0 m de tamanho, 0,25 mm de diâmetro interno e 0,25 $\mu \mathrm{m}$ de filme fino de cianopropil como fase estacionária) e um injetor com/sem divisão. As temperaturas do detector e do injetor foram de 250 e $230{ }^{\circ} \mathrm{C}$, respectivamente. O forno do CG-DIC foi programado para $65^{\circ} \mathrm{C}$ e mantido por 4 min., depois aquecido a $185{ }^{\circ} \mathrm{C}$ a $16{ }^{\circ} \mathrm{C}$ min. ${ }^{-1} \mathrm{e}$ mantido por 12 min., depois aquecido a $235^{\circ} \mathrm{C}$ a $20^{\circ} \mathrm{C}$ min. ${ }^{-1} \mathrm{e}$ mantido por 9 min. As vazões de gás utilizadas foram de $1,4 \mathrm{~mL} \mathrm{min.} .^{-1}$ para 
gás de arraste hidrogênio $\left(\mathrm{H}_{2}\right), 30 \mathrm{~mL} \mathrm{~m}^{-1}$ paragás de reposição nitrogênio $\left(\mathrm{N}_{2}\right)$ e 30 e $300 \mathrm{~mL} \mathrm{min.}{ }^{-1}$ para os gases de chama ( $\mathrm{H}_{2} \mathrm{e}$ ar sintético, respectivamente).

O modo de injeção dividida foi utilizado na proporção de 1:100 e o volume de injeções de amostra foi de 2,0 $\mu \mathrm{L}$. Foram identificadas EMAGs por comparação dos tempos de retenção dos constituintes da amostra com os dos padrões analíticos (mistura padrão EMAG, C4-C24, Saint Louis, Estados Unidos, Sigma-Aldrich). As áreas de pico foram determinadas usando o software ChromQuest ${ }^{\mathrm{TM}}$ 5.0, e as composições de ácidos graxos (AG) foram expressas como uma porcentagem relativa da AG total (Simionato et al., 2010). A análise foi realizada em triplicata.

\subsection{Análise estatística}

Os dados das análises de composição em ácidos graxos foram submetidos à análise de variância (ANOVA) e teste de Tukey ( $\mathrm{p}<0,05$ ), utilizando o software Assistat.

\section{Resultados e Discussão}

Na Tabela 1 abaixo encontram-se o somatório de ácidos graxos, e seus principais constituintes no leite humano, foi avaliado uma mãe realizando a coleta do leite sempre no mesmo horário do dia.

Tabela 1. Somatório de ácidos graxos presentes no leite humano e os seus principais constituintes.

\begin{tabular}{cccc}
\hline $\begin{array}{c}\text { Composição em Ácidos Graxos } \\
(\%)\end{array}$ & Colostro & Transição & Maduro \\
\hline IAGS & $39,11 \pm 1,48^{\mathrm{b}}$ & $43,94 \pm 0,84^{\mathrm{a}}$ & $45,46 \pm 0,22^{\mathrm{a}}$ \\
$16: 0$ & $20,94 \pm 0,79^{\mathrm{b}}$ & $24,42 \pm 1,41^{\mathrm{a}}$ & $20,54 \pm 0,47^{\mathrm{b}}$ \\
$\Sigma$ AGMI & $29,28 \pm 0,50^{\mathrm{ab}}$ & $29,92 \pm 0,31^{\mathrm{a}}$ & $28,82 \pm 0,42^{\mathrm{b}}$ \\
$18: 1 \mathrm{n}-9$ & $24,70 \pm 0,79^{\mathrm{a}}$ & $24,75 \pm 0,79^{\mathrm{a}}$ & $24,38 \pm 0,62^{\mathrm{a}}$ \\
AGPI & $31,61 \pm 1,87^{\mathrm{a}}$ & $26,14 \pm 0,54^{\mathrm{b}}$ & $25,72 \pm 0,43^{\mathrm{b}}$ \\
$18: 2 \mathrm{n} 6$ & $26,52 \pm 0,35^{\mathrm{a}}$ & $22,34 \pm 0,51^{\mathrm{b}}$ & $22,26 \pm 0,52^{\mathrm{b}}$ \\
$18: 3 \mathrm{n}-3$ & $1,71 \pm 0,37^{\mathrm{a}}$ & $1,12 \pm 0,10^{\mathrm{c}}$ & $1,57 \pm 0,05^{\mathrm{b}}$ \\
$20: 4 \mathrm{n}-6$ (AA) & $0,51 \pm 0,05^{\mathrm{a}}$ & $0,22 \pm 0,06^{\mathrm{b}}$ & $0,15 \pm 0,03^{\mathrm{b}}$ \\
$20: 5 \mathrm{n}-3$ (EPA) & $0,26 \pm 0,03^{\mathrm{ab}}$ & $0,32 \pm 0,03^{\mathrm{a}}$ & $0,17 \pm 0,02^{\mathrm{b}}$ \\
$22: 6 \mathrm{n}-3$ (DHA) & $0,44 \pm 0,08^{\mathrm{a}}$ & $0,30 \pm 0,05^{\mathrm{b}}$ & $00,19 \pm 0,01^{\mathrm{c}}$ \\
$\Sigma \mathrm{n}-6$ & $27,52 \pm 0,80^{\mathrm{a}}$ & $22,82 \pm 0,39^{\mathrm{b}}$ & $22,59 \pm 0,51^{\mathrm{b}}$ \\
$\Sigma \mathrm{n}-3$ & $4,11 \pm 0,29^{\mathrm{a}}$ & $3,33 \pm 0,20^{\mathrm{b}}$ & $3,24 \pm 0,08^{\mathrm{b}}$ \\
EPA+DHA & $0,70 \pm 0,10^{\mathrm{a}}$ & $0,62 \pm 0,12^{\mathrm{a}}$ & $0,36 \pm 0,03^{\mathrm{b}}$ \\
n-6/n-3 & $7,10 \pm 0,55^{\mathrm{a}}$ & $7,02 \pm 0,21^{\mathrm{a}}$ & $6,99 \pm 0,35^{\mathrm{a}}$ \\
AGPI/AGS & $0,81 \pm 0,08^{\mathrm{a}}$ & $0,59 \pm 0,02^{\mathrm{b}}$ & $0,57 \pm 0,01^{\mathrm{b}}$ \\
\hline
\end{tabular}

Resultados expressos em média \pm Desvio Padrão (DP) de triplicata. Valores com letras diferentes na mesma linha são significativamente diferentes $(\mathrm{p}<0,05)$ pelo teste de Tukey. (AGS - ácidos graxos saturados; AGMI - ácidos graxos monoinsaturados; AGPI - ácidos graxos poliinsaturados). Fonte: Autores (2021).

Avaliando os somatórios de ácidos graxos saturados (AGS) foi possível concluir que o estágio de lactação afeta esse somatório, pois, o colostro apresenta um menor valor quando comparado com o leite de transição e maduro, variando de 39,11 a 45,46\%. Dentre os ácidos graxos (AG) que constituem essa classe, temos como majoritário o ácido palmítico (16:0), sua concentração também é influenciada pelo estágio de lactação, sendo que seu valor variou de 20,54 a 24,42\%, Alves et al. (2021) e Manin et al. (2019) também encontraram 16:0 como o AGS de maior concentração. O 16:0 é um AG importante, pois 
ele é um ácido graxo que apresenta um efeito analgésico, melhorando assim o desconforto intestinal nos bebes (Demmelmair \& Koletzko, 2018).

Para o somatório de ácidos graxos monoinsaturados (AGMI) o mesmo foi observado, o estágio de lactação influencia nesse somatório, sendo que os valores encontrados estão entre 29,92 e 28,82\%. O ácido graxo encontrado em maior concentração para essa classe de AG é o ácido oleico (18:1n-9), esse fato corrobora com Alves et al. (2021) e Manin et al. (2019). É importante ressaltar que de todos os AG, não somente dentro da classe dos AGMI, o 18:1n-9 foi o encontrado em maior concentração no leite de transição, e maduro, já para o leite colostro o encontrado em maior concentração é o 18:2n-6.

O somatório de ácidos graxos poliinsaturados (AGPI) também apresentou diferença estatística quando comparado o estágio de lactação, sendo que o maior valor encontrado foi para o colostro, e os valores variaram de 31,61 a 25,72\%. Essa classe de AG é bastante importante para o desenvolvimento do bebê, pois é nela que encontramos os ácidos graxos essenciais e estritamente essenciais. O ácido graxo majoritário dessa classe é o ácido linoleico (18:2n-6), um ácido graxo estritamente essencial, ou seja, o ser humano não consegue sintetiza-lo, ou seja sua única fonte é a alimentação. Alves et al. (2021), Manin et al. (2019) e Manin et al. (2020) também encontraram esse sendo o majoritário para a classe de AGPI.

O 18:2n-6 foi encontrado com concentração entre 26,52 e $22,26 \%$, a fase de lactação também influenciou na concentração desse AG, o ácido linolênico (18:3n-3) é outro ácido graxo estritamente essencial, que apresentou uma variação entre a concentração dos estágios de lactação, com resultados entre 1,71 e 1,12\%. Esses AG também são importantes para o desenvolvimento do bebê, pois além de serem estritamente essenciais, são precursores dos ácidos graxos essenciais.

Os ácidos graxos essências são o ácido araquidônico (AA, 20:4n-6), o ácido eicosapentaenoico (EPA, 20:5n-3), e ácido docosahexaenóico (DHA, 22:6n-3), também são conhecidos como ácidos graxos poliinsaturados de cadeia longa, ambos são influenciados pelo estagio de lactação, sendo o DHA o que apresenta maior redução na sua concentração. Tanto o AA quanto EPA e DHA são AG imprescindíveis para o desenvolvimento do bebê, pois além do recém-nascido ter imaturidade fisiológica e não conseguir fazer a conversão dos ácidos graxos estritamente essências nos essências, eles são responsáveis pelo desenvolvimento cognitivo do bebê, visual, cerebral e imunológico (Rydlewski et al., 2020; Demmelmair \& Koletzko, 2018).

Ficou evidenciado analisando os principais ácidos graxos e somatórios que o leite de transição e maduro possuem semelhanças em suas concentrações, e que o leite colostro foi o que apresentou a composição mais distinta entre as três fases de lactação.

As razões de ômega 6/omega3 $(\Sigma \mathrm{n} 6 / \Sigma \mathrm{n} 3)$ e $\Sigma$ AGPI/ $/$ AGS foram realizadas com o intuito de atestar a qualidade lipídica do leite. Segundo a Organização Mundial da Saúde (2003), Simopoulos (2004) e Levy \& Tedstone (2017) as razões $\Sigma \mathrm{n} 6 / \Sigma \mathrm{n} 3$ devem estar entre 5 e 10, todos os valores encontrados estão dentro dessa faixa, variando de 6,99 a 7,10\%. Já a razão $\Sigma$ AGPI/ $\Sigma$ AGS deve apresentar valores acima de 0,45 , os valores encontrados também estão acima do valor estabelecido $(0,57$ a 0,81\%). Logo, com isso, as três fases do leite humano apresentam indicadores de boa qualidade lipídica, sendo isso de extrema importância para o desenvolvimento do bebê. Para a razão omega6/ômega3 a fase de lactação não apresentou diferença estatística, já para a razão AGPI/AGS apresentou diferença estatística, sendo que o colostro se difere das outras fases.

\section{Conclusão}

Como observado pela análise de ácidos graxos podemos concluir que a fase de lactação influencia diretamente na concentração dos principais ácidos graxos encontrados no leite humano, sendo que o leite de colostro é o que apresenta maior diferença entre as três fases de lactação, isso se deve ao fato de cada fase leite ser responsável por um momento na vida do bebê. Outro fator importante é que todos os ácidos graxos essenciais e estritamente essenciais foram encontrados e 
quantificados. Com isso novas pesquisas conduzidas de forma individualizadas devem ser realizadas, afim de atestar novas descobertas sobre a concentração de ácidos graxos no leite humano.

\section{Agradecimentos}

Os autores agradecem ao Conselho Nacional de Desenvolvimento Científico e Tecnológico (CNPq) e Coordenação de Aperfeiçoamento de Pessoal de Nível Superior (CAPES) pelo auxílio financeiro.

\section{Referências}

Alves, E. S., Castro, M. C., Saqueti, B. H., Manin, L. P., Silveira, R. D., Souza, P. M., \& Visentainer, J. V. (2021). Whey Isolation from Rejected Human Milk and Its Lipid Content Characterization by GC-FID and ESI-MS. Journal of the Brazilian Chemical Society, 32, $1884-1894$.

Ballard, O., \& Morrow, A. L. (2013). Human milk composition: nutrients and bioactive factors. Pediatric Clinics, 60, 49-74.

Binns, C., Lee, M., \& Low, W. Y. (2016). The long-term public health benefits of breastfeeding. Asia Pacific Journal of Public Health, $28,7-14$.

Bourlieu, C., \& Michalski, M. C. (2015). Structure-function relationship of the milk fat globule. Current Opinion in Clinical Nutrition \& Metabolic Care, 18, $118-127$.

Demmelmair, H., \& Koletzko, B. (2018). Lipids in human milk. Best Practice \& Research Clinical Endocrinology \& Metabolism, $32,57-68$.

Folch, J., Lees, M., \& Stanley, G. S. (1957). A simple method for the isolation and purification of total lipides from animal tissues. Journal of biological chemistry, 226, 497-509.

Gurnida, D. A., Rowan, A. M., Idjradinata, P., Muchtadi, D., \& Sekarwana, N. (2012). Association of complex lipids containing gangliosides with cognitive development of 6-month-old infants. Early human development, 88, 595-601.

International Organization for Standardization - ISO. 12966:2017. Animal and vegetable fats and oils - Gas chromatography of fatty acid methyl esters Part 2: Preparation of methyl esters of fatty acids. https://www.iso.org/obp/ui/\#iso:std:iso:12966:-2:ed-2:v1:en

Johnston, M., Landers, S., Noble, L., Szucs, K., \& Viehmann, L. (2012). Breastfeeding and the use of human milk. Pediatrics, 129, e827-e841

Levy, L., \& Tedstone, A. (2017). UK dietary policy for the prevention of cardiovascular disease. In Healthcare. Multidisciplinary Digital Publishing Institut. 5,9 .

Manin, L. P., Rydlewski, A. A., Galuch, M. B., Pizzo, J. S., Zappielo, C. D., Senes, C. E., \& Visentainer, J. V. (2019). Evaluation of the lipid quality of lyophilized pasteurized human milk for six months by GC-FID and ESI-MS. Journal of the Brazilian Chemical Society, 30, $1579-1586$.

Manin, L. P., Rydlewski, A. A., Alves, E. S., Ponhozi, I. B., Castro, M. C., Saqueti, B. H. F., \& Visentainer, J. V. (2020). Evaluation of the lipid composition of the three lactation phases of raw, pasteurized and lyophilized pasteurized human milk. Research, Society and Development, 9, e26891211136e26891211136.

Mehrotra, V., Sehgal, S. K., \& Bangale, N. R. (2019). Fat structure and composition in human milk and infant formulas: Implications in infant health. Clinical Epidemiology and Global Health, 7, 153-159.

Organização Mundial da Saúde. (2003). Global Strategy on Diet, Physical Activity and Health: Diet, Nutrition and the Prevention of Chronic Diseases. https://www.who.int/dietphysicalactivity/publications/trs916/en/gsfao_overall.pdf?ua=1

Pereira, A. S., Shitsuka, D. M., Parreira, F. J., \& Shitsuka, R. (2018). Metodologia da pesquisa científica. UFSM. https://repositorio.ufsm.br/bitstrea $\mathrm{m} /$ handle/1/15824/Lic_Computacao_Metodologia-Pesquisa-Cientifica.pdf?sequence=1.

Prell, C., \& Koletzko, B. (2016). Breastfeeding and complementary feeding: recommendations on infant nutrition. Deutsches Ärzteblatt International, 113, 435 .

Rydlewski, A. A., Pizzo, J. S., Manin, L. P., Zappielo, C. D., Galuch, M. B., Santos, O. O., \& Visentainer, J. V. (2020). Métodos analíticos utilizados para a determinação de lipídios em leite humano: uma revisão. Revista Virtual de Química, 12.

Rydlewski, A. A., Silva, P. D., Manin, L. P., Tavares, C. B., Paula, M. G., Figueiredo, I. L., \& Visentainer, J. V. (2019). Lipid Profile Determination by Direct Infusion ESI-MS and Fatty Acid Composition by GC-FID in Human Milk Pools by Folch and Creamatocrit Methods. Journal of the Brazilian Chemical Society, 30, 1063-1073.

Simionato, J. I., Garcia, J. C., Santos, G. T. D., Oliveira, C. C., Visentainer, J. V., \& Souza, N. E. D. (2010). Validation of the determination of fatty acids in milk by gas chromatography. Journal of the Brazilian Chemical Society, 21, 520-524.

Simopoulos, A. P. (2004). Omega-6/omega-3 essential fatty acid ratio and chronic diseases. Food reviews international, $20,77-90$.

Van Gysel, M., Cossey, V., Fieuws, S., \& Schuermans, A. (2012). Impact of pasteurization on the antibacterial properties of human milk. European journal of pediatrics, 171, 1231-1237. 
Research, Society and Development, v. 10, n. 14, e308101422174, 2021

(CC BY 4.0) | ISSN 2525-3409 | DOI: http://dx.doi.org/10.33448/rsd-v10i14.22174

Vyssotski, M., Bloor, S. J., Lagutin, K., Wong, H., \& Williams, D. B. G. (2015). Efficient separation and analysis of triacylglycerols: quantitation of $\beta$ palmitate (OPO) in oils and infant formulas. Journal of agricultural and food chemistry, 63, 5985-5992.

Wang, L., Li, X., Liu, L., da Zhang, H., Zhang, Y., Chang, Y. H., \& Zhu, Q. P. (2020). Comparative lipidomics analysis of human, bovine and caprine milk by UHPLC-Q-TOF-MS. Food chemistry, 310, 125865.

Zhao, P., Zhang, S., Liu, L., Pang, X., Yang, Y., Lu, J., \& Lv, J. (2018). Differences in the triacylglycerol and fatty acid compositions of human colostrum and mature milk. Journal of agricultural and food chemistry, 66, 4571-4579. 